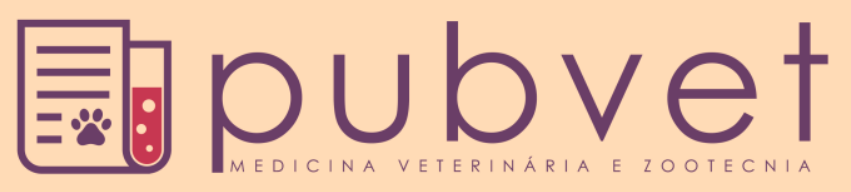

https://doi.org/10.22256/pubvet.v12n6a101.1-9

\title{
Alterações comportamentais e fisiológicas em cães detectores sob influência do estresse de confinamento
}

\author{
Lara Lívia Munique Machado ${ }^{1 *}$, Júlio César Montanha ${ }^{\ominus 2}$, Sérgio Leme da Silva ${ }^{\circledR}$ \\ ${ }^{I}$ Departamento de Processos Psicológicos Básicos - PPB/Instituto de Psicologia-IP/Universidade de Brasilia-UnB, BrasiliaDF-Brasil, laraliviamachado@gmail.com \\ ${ }^{2}$ Departamento de Processos Psicológicos Básicos - PPB/Instituto de Psicologia - IP/Universidade de Brasilia-UnB, BrasiliaDF-Brasil, montanhabio@gmail.com \\ ${ }^{3}$ Departamento de Psicologia, Universidade Federal de São Carlos-UFSCar, São Carlos/SP-Brasil e Laboratório de Psicobiologia, Instituto de Psicologia, \\ UnB, Brasília/DF-Brasil, lemesergio@yahoo.com.br
}

\begin{abstract}
RESUMO. Este trabalho foi realizado para estudar os efeitos fisiológicos e comportamentais no desempenho olfativo de cães policiais, após situações cotidianas de estresse que ocorriam previamente à execução da tarefa de detecção de drogas e explosivos. O efeito do estresse foi analisado a partir da simulação de confinamento desses animais até o local da detecção de drogas ou explosivos, em diferentes tempos de transporte, utilizando três tipos de confinamento em caixas de transporte, 30 minutos (30") 3 horas e 30 minutos (03'30") e 5 horas e 30 minutos (05'30"). Para avaliar os níveis de cortisol impactados das condições de estresse (confinamentos) nos indivíduos do estudo foi necessário fazer coletas não-invasivas de saliva. Este procedimento se apresenta eficiente, no caso de cães, pois é de fácil utilização e não provoca alterações significativas no estado fisiológico dos animais. Os resultados apresentados demonstraram o aparecimento do estresse e alterações comportamentais, indicando uma diminuição do desempenho olfativo de cães detectores de drogas e explosivos.
\end{abstract}

Palavras chave: bem-estar, cães policiais, desempenho, drogas e explosivos, estresse

\section{Behavioral and physiological changes in detection dogs under influence of confinement stress}

ABSTRACT. The present article aimed to study the physiological and behavioral effects on
the olfactory performance of police dogs, after everyday stress situations that occurred before
to the execution of the task of detecting drugs and explosives. The effect of stress was studied
from the simulation of confinement of these animals to the detection of drugs or explosives, at
different transport times, using three types of confinement in transport boxes, 30 minutes ( 30
minutes), 3 hours and 30 minutes ( 03 ' 30 ") and 5 hours and 30 minutes ( $05^{\prime} 30$ "). In order to
evaluate the affected cortisol levels of the stress conditions (confinement) in the study subjects,
it was necessary to make noninvasive saliva collections. This procedure is efficient in the case
of dogs, since it is easy to use and does not cause significant changes in the physiological state
of the investigated dogs. The results presented demonstrated the appearance of stress and
behavioral changes, indicating a decrease in the olfactory performance of drug detecting dogs
and explosives.

Key words: polices dogs, drugs and explosives, welfare, performance, stress

\section{Alteraciones conductuales y fisiológicas en perros detectores bajo la influencia del estrés de confinamiento}

RESUMEN. El presente artículo fue realizado para estudiar los efectos fisiológicos y de comportamiento en el desempeño olfativo de perros policiales, después de situaciones cotidianas de estrés que ocurrían previamente a la ejecución de la tarea de detección de 
drogas y explosivos. El efecto del estrés fue analizado a partir de la simulación de confinamiento de esos animales hasta el lugar de la detección de drogas o explosivos, en diferentes tiempos de transporte, utilizando tres tipos de confinamiento en cajas de transporte, 30 minutos (30") 3 horas y 30 minutos (03'30 ") y 5 horas y 30 minutos (05'30"). Para evaluar los niveles de cortisol impactados de las condiciones de estrés (confinamientos) en los individuos del estudio fue necesario hacer colectas no invasivas de saliva. Este procedimiento se presenta eficiente, en el caso de perros, pues es de fácil utilización y no provoca alteraciones significativas en el estado fisiológico de los animales. Los resultados presentados demostraron la aparición del estrés y alteraciones comportamentales, indicando una disminución del desempeño olfativo de perros detectores de drogas y explosivos.

Palabras clave: Bienestar, perros policiales, rendimiento, drogas y explosivos, estrés

\section{Introdução}

A relação cooperativa entre seres humanos $\mathrm{e}$ cães (Canis familiaris) existe a mais de $140 \mathrm{mil}$ anos (Moody et al. 2006), mas a utilização de suas habilidades de trabalho começaram a se aperfeiçoar nos últimos 10 mil anos (Lindsay 2000). Durante esse período, os animais passaram pela domesticação, um intrincado processo que envolveu alterações genéticas e uma seleção rigorosa das raças (Lindsay 2000).

A mobilização em torno da melhora sucessiva da espécie para um melhor desempenho no trabalho trouxe os problemas relacionados aos níveis de bem-estar físico e psicológico (Broom \& Johnson 1993; Schatz \& Palme 2001), diretamente ligados aos transtornos de comportamento canino (Santana et al. 2010).

O estresse ambiental e fisiológico está entre os fatores que mais influenciam na fisiologia do sistema olfativo (Gazit \& Terkel 2003; Ahrens et al. 2005; Haverbeke et al. 2008). Sua cascata de resposta biológica está intimamente ligada ao sistema límbico (Wilson et al. 2004) que é o responsável pelas respostas relacionadas às emoções (LeDoux 2003), tais quais, nas reações de "luta ou fuga". Assim, podemos pressupor que qualquer alteração ambiental e/ou orgânica pode levar ao comprometimento significativo da performance olfativa do indivíduo (Strasser et al. 1993; Gazit \& Terkel 2003).

O cortisol é o hormônio ligado diretamente a situações de estresse (Broom \& Johnson 1993; Beerda et al. 1996; Haverbeke et al. 2008). Devido a sua importância na manutenção da homeostase orgânica, o cortisol deve ser considerado uma medida importante quando abordamos as condições de bem-estar animal e suas respostas aos diferentes desafios ambientais e físicos (Ahrens et al. 2005; Haubenhofer \& Kirchengast 2006). Os níveis de cortisol salivar se comparam aos níveis de cortisol plasmático (Haubenhofer \& Kirchengast 2006; Montanha et al. 2009), apresentando as mesmas taxas de secreção, não havendo diferenças entre suas mensurações. O que se observa é um "delay" de aproximadamente 4 minutos entre o aumento do cortisol plasmático e um sequente incremento nos níveis de cortisol salivar. Havendo uma coleta em tempo menor que 4 minutos, podemos diminuir os prováveis efeitos estressantes causados pela coleta (Haubenhofer \& Kirchengast 2006).

Para medir os níveis de cortisol e estabelecer as condições de estresse nos indivíduos do estudo é necessário fazer coletas não-invasivas de saliva (Montanha et al. 2009). Este procedimento se apresenta eficiente, no caso de cães, pois é de fácil utilização e não provoca alterações significativas no estado fisiológico dos investigados (Beerda et al. 1996; Haubenhofer \& Kirchengast 2006).

Cães a serviço da polícia ou dos bombeiros passam por situações consideradas estressoras que podem modificar seu estado de equilíbrio físico e psicológico (Bergeron et al. 2002; Ahrens et al. 2005). Dentre esses fatores o transporte terrestre, o transporte aéreo (Bergeron et al. 2002), o tempo de confinamento, a quantidade de treinamento e o confinamento em caixas de transporte são as situações mais comuns em seu cotidiano.

Determinar os níveis de estresse pelas quais esses cães passam é de extrema importância para manutenção dos níveis de bem-estar e, consequentemente, o aumento na performance olfativa durante o trabalho (Gazit \& Terkel 2003). Além disso, estudos sobre o faro canino foram desenvolvidos com o propósito de explorar a sua intrincada fisiologia e plasticidade, aproveitandose ao máximo daquilo que é oferecido por cada animal (Gazit \& Terkel 2003). Fatos que trouxeram para a área de segurança, avanços na detecção de drogas (Adams \& Johnson 1994; 
Jezierski et al. 2008) explosivos (Lovett 1992); na busca por pessoas desaparecidas e vítimas de soterramento (Morrison 2000), dentre outras (Gazit \& Terkel 2003).

Assim, procurou-se definir qual é a zona de conforto em olfação e quais são os elementos estressores que diminuem a eficiência olfativa (Gazit \& Terkel 2003). Um novo horizonte a respeito das aptidões caninas começa a se formar. $\mathrm{O}$ animal não será mais visto como uma máquina, um sistema estanque, mas sim como conjunto de informações morfológicas, fisiológicas e comportamentais que devem ser avaliadas constantemente.

Quando vamos iniciar o estudo a respeito de uma espécie, devemos ter em mente que o indivíduo analisado não é um sistema inerte e fechado, mas sim um somatório de eventos que possibilitam ter um exame mais amplo e completo (Bateson et al. 2004). Essa definição permite entender que processos biológicos são compostos por eventos fisiológicos e psicológicos, não havendo exclusão entre eles, mas sim uma sobreposição com fortes correlações (Beerda et al. 1996; Haverbeke et al. 2008; Montanha et al. 2009).

\section{Material e Métodos}

Os 6 (seis) animais submetidos ao estudo foram cães (Canis lupus familiaris) das raças Pastor Alemão (2 cães) e Mallinois - ou Pastor Belga (4 cães), do Canil Central do Departamento de Polícia Federal (CC - DPF), com sede em Brasília - DF, com idades entre 3 e 4 anos.

Os cães do CC - DPF vivem em canis individuais, de onde são retirados para liberdade em horário matinal, no qual o cão é solto para brincar e realizar suas necessidades biológicas, treinamentos diários e trabalho de detecção. Todos os seis animais disponibilizados para a estudo são utilizados pela Polícia Federal no serviço de segurança nacional do Brasil. Recebendo, para tanto, treinamento profissionalizado e padronizado pelas exigências do CC - DPF para a realização de busca e detecção de odores alusivos aos cheiros exalados por drogas, explosivos e seus insumos produzidos ou comercializados no país. E esses animais ainda estavam com o mesmo nível de condicionamento referentes ao aprendizado das respostas de detecção frente ao estímulo olfativo da droga e explosivo, fornecido pelos treinamentos pelo CC - DPF. Nível este que considera o cão apto para o trabalho, sendo, para tanto, todos devidamente certificados para este fim. Durante o estudo todos os animais participaram de todas as condições experimentais, em condições semelhantes, sendo, para tanto, cada animal controle de si mesmo.

Para que houvesse a simulação de situações do estresse sobre o confinamento para o transporte de cunho operacional dos animais, foram utilizadas caixas de transporte animal (Figura 1), já utilizadas pelo CC - PF em suas atividades de treinamento e deslocamento.

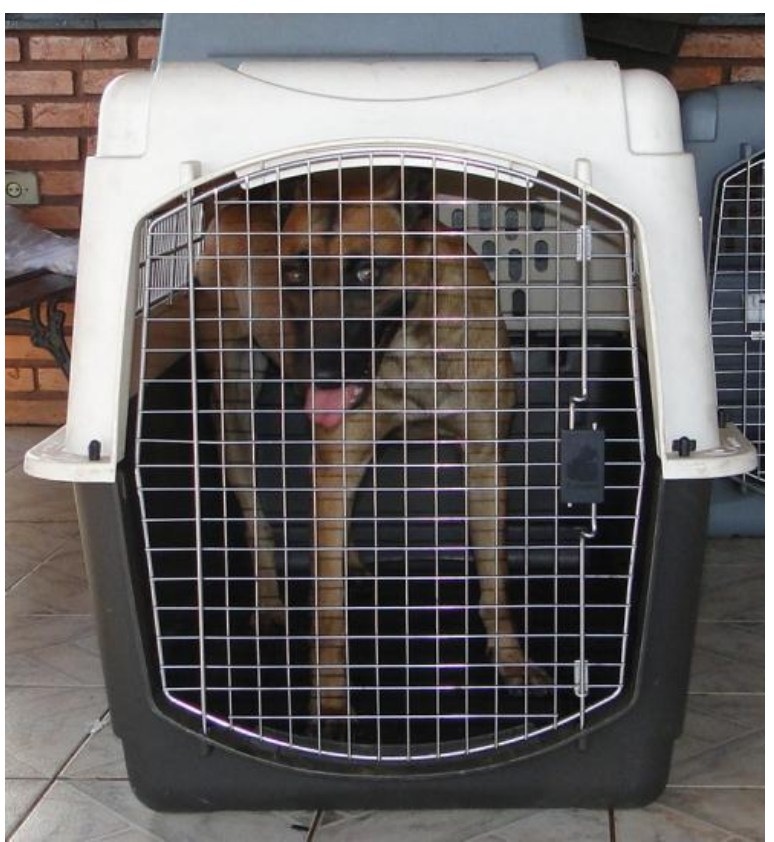

Figura 1. Caixa de transporte

$\mathrm{Na}$ primeira fase dos trabalhos foram realizadas as simulações dos confinamentos em caixas de transporte (Figura 1) e em cabines de transporte para cães em viaturas (Figura 2), ambas utilizadas para a simulação de transporte dos animais do CC - PF até o local de realização de atividades de busca e detecção.

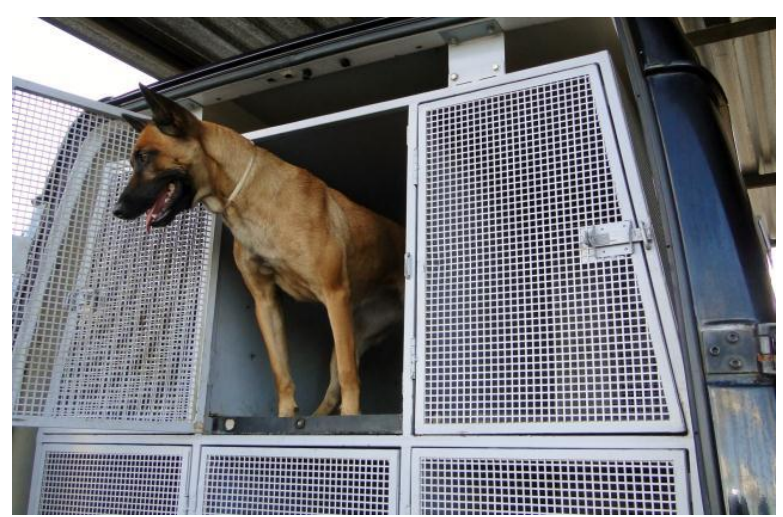

Figura 2. Cabines de transporte 
Foram definidos três roteiros de transporte para caracterizar as variáveis fixas dos tempos de confinamento em caixas de transporte correspondentes às viagens de curta, média e longa distância (Tabela 1).

Tabela 1. Roteiros de transporte

\begin{tabular}{lccc}
\hline Roteiro & Roteiro 1 & Roteiro 2 & Roteiro 3 \\
\hline Tempo de & Meia hora & 3 horas e & 5 horas e \\
confinamento & $(30$ ) $)$ & $\begin{array}{c}30 \text { minutos } \\
\left(30^{\prime}\right)\end{array}$ & $\begin{array}{c}30 \text { minutos } \\
\left(5^{\prime} 30^{\prime \prime}\right)\end{array}$ \\
\hline
\end{tabular}

O roteiro 1, possui um tempo de confinamento nas cabines de transporte da viatura, apenas para o transporte dos cães do canil até o local de desempenho das atividades de busca e detecção, sendo, portanto, considerada como 30 minutos (30") de confinamento em caixas de transporte, ou seja, o animal foi transportado, via terrestre, diretamente do canil, em viaturas próprias, para o local de desempenho das tarefas de busca e detecção. $\mathrm{O}$ roteiro 2 apresentou um tempo total de 3 horas e 30 minutos (03'30") de confinamento nas caixas de transporte e cabine, considerado para trechos de média distância. E o roteiro 3 apresentou um tempo total de 5 horas e 30 minutos (05'30") de confinamento, considerado para trechos de longas distâncias.

Os animais foram levados para a área de depósito da Polícia Federal, onde permaneceram confinados em suas respectivas caixas de transporte de acordo com cada condição de rotina das atividades propostas, em situações idênticas de temperatura ambiente, umidade relativa do ar, incidência de luz e efeitos acústicos. Todos os 6 (seis) animais participaram de todas as condições de confinamento e transporte, conforme a tabela de distribuição randômica, repetindo três vezes cada rotina de confinamento para cada animal, distribuídas em 9 (nove) semanas (Tabela 2). Essa distribuição visa controlar o efeito da habituação do cão, no que diz respeito a níveis de estresse e eficiência de olfação, devido à repetição continua de uma mesma rotina.

Tabela 2. Distribuição dos sujeitos de acordo com as rotinas de confinamento

\begin{tabular}{|c|c|c|c|}
\hline \multirow[b]{2}{*}{ Todos os sujeitos } & \multicolumn{3}{|c|}{$\begin{array}{l}\text { Roteiros de confinamento em caixas } \\
\text { de transporte }\end{array}$} \\
\hline & $\begin{array}{l}\text { Roteiro } 1 \\
(30 ")\end{array}$ & $\begin{array}{l}\text { Roteiro } 2 \\
(3 \text { '30') }\end{array}$ & $\begin{array}{c}\text { Roteiro } 3 \\
\left(50^{\prime \prime}\right)\end{array}$ \\
\hline Semanas & $1^{\mathrm{a}}, 6^{\mathrm{a}}$ e $9^{\mathrm{a}}$ & $2^{a}, 5^{a}$ e $7^{a}$ & $3^{a}, 4^{a}$ e $8^{a}$ \\
\hline
\end{tabular}

Durante as atividades de detecção, foram feitas as avaliações de desempenho de farejamento por simulações de busca e detecção dos odores de drogas e explosivos, onde eram observadas as categorias comportamentais estabelecidas para o presente estudo (Freitas \& Nishida 2007) e aferidas as concentrações de cortisol salivar.

A tarefa foi realizada utilizando 5 (cinco) salas de aula denominadas em A, B, C, D e E, de dimensões semelhantes $(10$ metros de comprimento e 8 de largura) dispostas sequencialmente conforme distribuição aleatória das mesmas para cada passagem, nas quais o animal entrava uma única vez em cada sala para realizar a atividade de busca e detecção, sendo premiado com brinquedos a cada indicação correta da localização do item de busca (droga ou explosivo) (Figura 3).

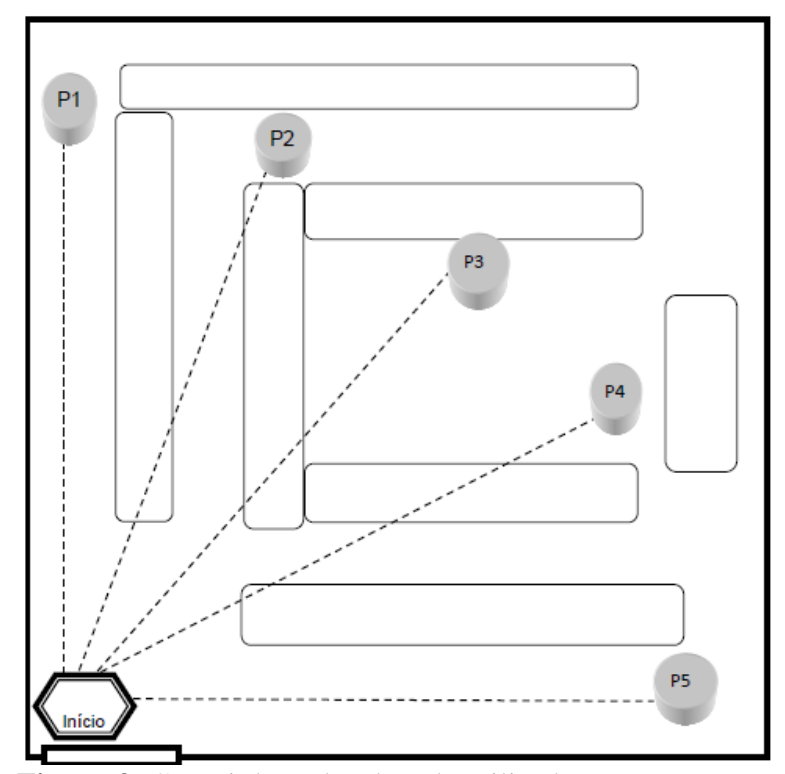

Figura 3. Croqui das salas de aula utilizadas com os Pontos de Detecção (PD1, PD2, PD3, PD4 e PD5)

Em cada sala de aula havia 5 (cinco) suportes circulares de concreto com dimensões iguais, os quais demarcavam os Pontos de Detecção (PD) equidistantes do ponto inicial de partida do cão, tendo cada ponto de detecção uma distância de 6,70 metros do ponto inicial de partida do cão. Nos suportes de concreto eram alocados potinhos plásticos contendo os odores de substâncias distintas, drogas ou explosivos, dependendo do tipo de detecção que o animal estava treinado a realizar.

As substâncias ilícitas e os explosivos utilizados na pesquisa são de manipulação e controle exclusivo da Polícia Federal, sendo o trabalho, portanto, acompanhado por um agente 
autorizado da Polícia Federal do Brasil e, para tanto, deixamos nossos agradecimentos à equipe do Canil Central da polícia Federal do Brasil e em oportuno aos agentes Miranda, Nelson, Marcelo e Cláudio que participaram ativamente do projeto.

A tarefa foi realizada com o cão partindo da entrada da sala (Início) para seu interior onde se executou a detecção dos itens de busca (drogas ou explosivos) sem a presença do operador. Desta forma, evitou-se o efeito da presença do condutor durante o farejamento (Lefebvre et al. 2007; Lit et al. 2011).

Em cada sala de tarefas e em todas as passagens de busca foram registrados os tempos totais gastos no farejamento, bem como o repertório comportamental durante as atividades. O tempo foi considerado para aferir a eficiência e o desempenho em olfação e os comportamentos registrados durante as atividades, para delinear o padrão requerido nas atividades realizadas pelo CC-DPF. Essas variáveis ainda foram relacionadas com o nível de estresse aferido pelos índices de cortisol salivar, no sentido de confirmar o estado psicofisiológico do animal, relacionado aos padrões comportamentais e o desempenho olfativo.

As variáveis: 1) tempo de busca, 2) padrão de farejamento, 3) foco de detecção, 4) perfil de busca e 5) perfil de ambulação, foram registradas durante o desenvolvimento das tarefas. O objetivo da mensuração dessas variáveis foi o de levantar os principais comportamentos apresentados pelo animal durante as atividades de busca e detecção, verificando o efeito do estresse do confinamento em caixas de transporte sobre o desempenho olfativo dos cães.

Durante todas as atividades de confinamento e detecção, os cães foram aferidos em seus níveis de cortisol salivar, através de coletas não-invasivas (Figura 4).

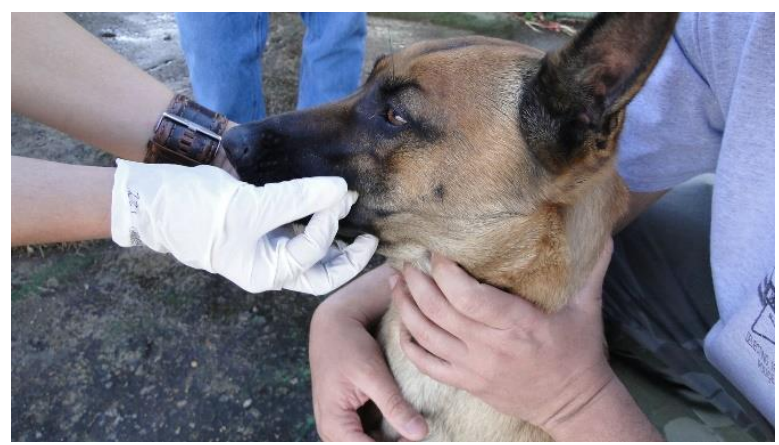

Figura 4. Coleta salivar não-invasiva

As coletas salivares foram realizadas em três momentos distintos: antes do confinamento, após o confinamento e após a detecção (Tabela 3).

Desta forma, foram estabelecidas as concentrações de cortisol salivar nas diferentes rotinas de confinamento em caixas de transporte e nos diferentes momentos de coleta, determinados pela presença ou não de confinamento e o início e fim das atividades de busca e detecção.

A coleta salivar foi feita através de coletor do tipo Salivette ${ }^{\circledR}$, via oral, sem a possibilidade de retenção por parte do animal ou contaminação do mesmo por sangue ou sujeira. O coletor Salivette ${ }^{\circledR}$ é composto por dois cilindros plásticos e um coletor de algodão em forma de cilindro. $\mathrm{O}$ cilindro interno de plástico é usado como depósito do coletor de algodão e o externo como depósito do material biológico separado do coletor de algodão após centrifugação. $\mathrm{O}$ cilindro de algodão foi levemente passado na cavidade oral do animal, no assoalho da região vestibular da boca, mantendo-o posicionado por pelo menos um minuto, até sua parcial umidificação, conforme treinamento técnico da equipe de pesquisadores para coleta, na fase de testes do projeto.

Tabela 3. Descrição do momento das coletas salivares

\begin{tabular}{lll}
\hline Referência da coleta & Nome/ Abreviatura & Descrição \\
\hline $\begin{array}{l}\text { Referente ao momento antes } \\
\text { de qualquer atividade observada }\end{array}$ & Antes do confinamento & $\begin{array}{l}\text { Logo após o período de liberdade do canil, por } \\
\text { volta das 7 horas da manhã, antes do confinamento } \\
\text { em caixas de transporte e das tarefas de busca e } \\
\text { detecção. }\end{array}$ \\
\hline $\begin{array}{l}\text { Referente ao momento do com- } \\
\text { finamento nas caixas de transporte }\end{array}$ & Durante o confinamento & $\begin{array}{l}\text { Imediatamente após a retirada dos animais do } \\
\text { confinamento, que coincide com o momento antes } \\
\text { do início das tarefas de busca e detecção. }\end{array}$ \\
\hline $\begin{array}{l}\text { Referente às tarefas de busca e } \\
\text { detecção a serem realizadas }\end{array}$ & Após o confinamento & $\begin{array}{l}\text { Logo após a realização das atividades de busca e } \\
\text { detecção. }\end{array}$ \\
\hline
\end{tabular}


Após a coleta, as amostras de cortisol salivar foram congeladas a temperatura de $-2{ }^{\circ} \mathrm{C} \mathrm{a}-3{ }^{\circ} \mathrm{C}$ até serem descongeladas para posterior centrifugação na velocidade de $5000 \mathrm{rpm}$ durante 3 minutos, onde a saliva era separada do algodão ficando depositada nos tubos Salivette ${ }^{\circledR}$. A partir de então, foram recongeladas pelo período de 8 meses e submetidas ao laboratório para devidas análises via radio-imoimunoensaio (RIA) conforme realizado em Montanha et al. (2009).

$\mathrm{O}$ princípio bioético do presente projeto atrela o uso do capital financeiro do mesmo mediante a aprovação do presente projeto no Comitê de Ética do Uso Animal do Instituto de Ciências Biológicas da Universidade de Brasília sob a referência UnBDOC no 42544/2012, cuja cópia está disponibilizada no Apêndice I deste trabalho

\section{Resultados}

O resultado para os níveis de cortisol salivar, usando o teste de hipótese de Friedman para amostras relacionadas e análise de variância por ranqueamento demonstrou diferenças entre os níveis de cortisol medidos antes do confinamento (média ranqueada $=1,58$ ) e após o confinamento (média ranqueada $=2,96$ ), e entre os níveis aferidos após o confinamento e após a detecção (média ranqueada $=1,46$ ) com valores de teste estatístico iguais a $-1,375$ e 1,500 e valores de $p=$ 0,002 e 0,001 , respectivamente (Figura 5).

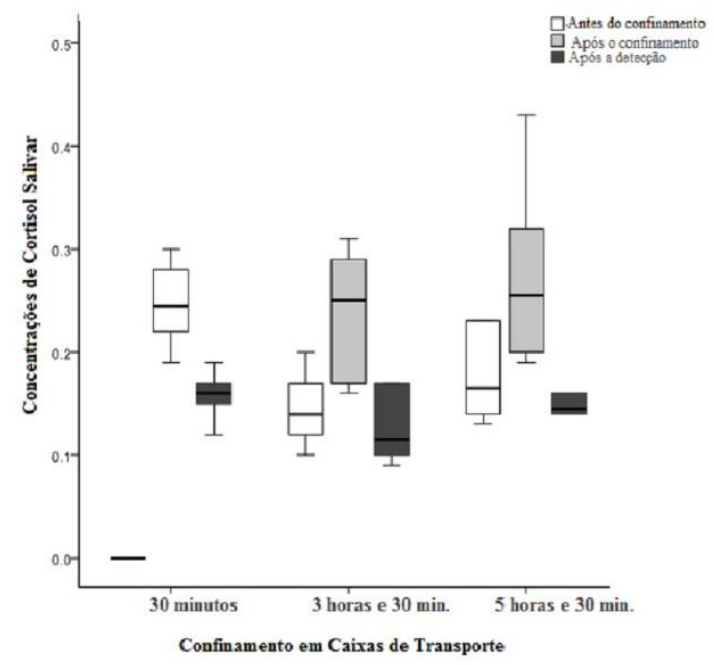

Figura 5. Níveis de cortisol salivar nos momentos de coleta (antes das atividades, após o confinamento e após a detecção) nos três tipos de confinamento com (30', 3'30" e 5'30") com significância $(\mathrm{P}<0,05)$.

A relação do tempo de confinamento em caixas de transporte com o tempo de busca do objeto apresentou-se positiva, indicando que quanto maior o tempo de confinamento nas caixas de transporte, maior foi o tempo de busca do animal para encontrar o item de busca (droga ou explosivo), com estimativa do parâmetro igual a 3,91 e erro médio de $0,98(\mathrm{P}<0,001)$.

A relação do tempo de confinamento em caixas de transporte com o padrão de farejamento de boca aberta $(-0,89 \pm 1,18 ; \mathrm{P}<0,001)$ apresentou-se negativa, indicando que quanto maior foi o tempo de confinamento menor foi o tempo que o cão farejou com a boca aberta.

Para as demais categorias de comportamentos não foram verificadas respostas significativas ao efeito do tempo de confinamento em caixas de transporte decorrentes da necessidade de transporte.

As regressões de Poisson analisadas para as categorias de foco (consideradas como variáveis de contagem) mostraram que o tipo de confinamento influenciou no comportamento de emissão de respostas de falso positivo sentado com contato visual $(9,60 \pm 0,71 ; \mathrm{P}<0,001)$, sugerindo que quanto maior o tempo de confinamento em caixas de transporte, maior a probabilidade do surgimento desse comportamento.

Pela distribuição de frequências para as subcategorias de acertos e erros (falsos positivos) do foco de detecção vemos uma tendência do aparecimento de maior ocorrência de erros no confinamento de 5,5 horas (Figura 6).

As diferenças de raça, idade e sexo não influenciaram nos resultados obtidos uma vez que todos os sujeitos eram controles de si mesmos, não sendo, portanto, os resultados para cada sujeito comparados com os demais.

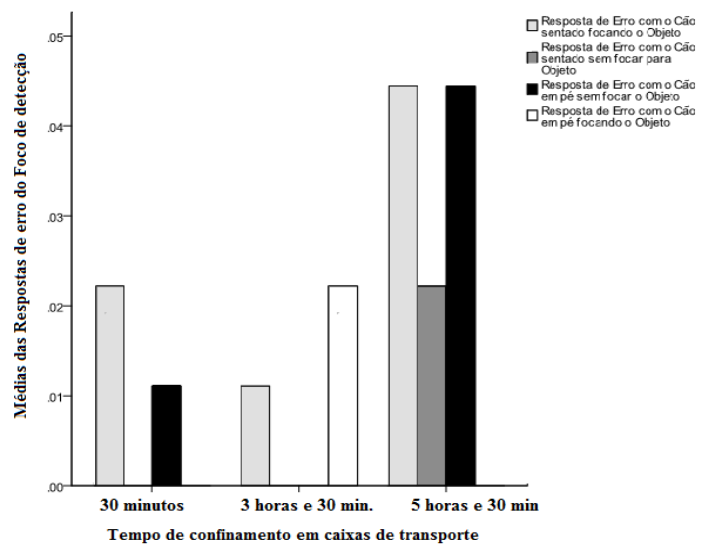

Figura 6. Médias de respostas de erros (falsos positivos) no foco na detecção em cada tempo de confinamento durantes as atividades de busca e detecção com significância $(P<0,05)$. 


\section{Discussão}

O presente projeto, desde sua concepção, levantou uma discussão ampla a respeito das condições psicológicas, fisiológicas e éticas pelas quais passam os cães de serviço de detecção de drogas e explosivos do Canil Central do Departamento de Polícia Federal (CC/DPF), com foco em investigar as influências de fatores estressantes como o confinamento em caixas de transporte, decorrentes das necessidades de transporte desses animais para a realização dos trabalhos de busca e detecção de drogas e explosivos, no desempenho olfativo durante o trabalho, por todo o Brasil.

Os resultados apresentados pelas relações de concentração de cortisol e as rotinas de coleta nos diferentes tempos de confinamento nas caixas apresentaram-se significativos, com valores de $p$ $<0,05$, para as relações das concentrações de cortisol avaliadas antes do confinamento e após o confinamento, e entre as concentrações de cortisol salivar medidas após o confinamento e após as detecções. Pode-se afirmar então, que o confinamento em caixas de transporte é um fator relevante para o aumento dos níveis de cortisol nos tempos determinados para as rotinas 2 e 3 de confinamento em caixas de transporte, demostrando que o confinamento de 3 horas já é suficiente para o aparecimento de concentrações mais altas de cortisol.

Posteriormente percebemos que as concentrações de cortisol diminuem, após a realização das tarefas de detecção e que no tipo de confinamento do roteiro 3 (5'30") prevalecem os níveis mais altos de concentração de cortisol, indicando que quanto maior o tempo de confinamento maior as concentrações de cortisol e que o trabalho de busca e detecção provavelmente funcione como um promotor de bem-estar e equilíbrio do cão.

Nossos resultados demonstram que o aumento do tempo do confinamento para o transporte faz com que o tempo de busca dos itens, drogas e explosivos, também aumente. Isso possivelmente significa que as condições de confinamento e transporte tendem a causar um aumento nas concentrações de cortisol, alterando a condição fisiológica do animal que de alguma forma, ainda não conhecida, afete a eficiência olfativa do cão $\mathrm{e}$, consequentemente, requer do animal mais tempo para a realização da atividade de busca e detecção.
O mesmo se verifica para o perfil de busca. Quanto maior o tempo de confinamento nas caixas de transporte, menor é a capacidade de identificação dos pontos de detecção, visto que os cães apresentavam perfil de busca cada vez mais global com o aumento do tempo de confinamento nas caixas. Ou seja, o animal passa a aumentar o seu campo de busca, farejando distintos pontos da sala, na medida em que se aumenta o tempo de confinamento. Isso pode significar, na rotina, um possível aumento do espaço explorado, maior contato com uma variabilidade de odores, não conseguindo distinguir o odor do item de busca, levando ao maior gasto de tempo para efetuar o trabalho e aumentando as chances de uma indicação errada (falso positivo).

O comprometimento olfativo pode estar relacionado com os níveis de estresse fisiológico sofrido pelos animais durante as rotinas de confinamento, como demostrado em nosso estudo e corroborando com outros estudos em cães, os quais apontam que o estresse do confinamento em transportes prejudica o desempenho olfativo (Bergeron et al. 2002).

Os resultados obtidos no presente estudo corroboraram as hipóteses iniciais quando obteve respostas de parâmetros comportamentais e fisiológicos, cujas quais se pode concluir que o tempo de confinamento para o transporte em caixas de transporte é fator estressante e consequentemente influência de certa maneira na eficiência olfativa do cão, retardando ou diminuindo seu desempenho nos trabalhos de busca e detecção de drogas e explosivos. Assim, pela relevância e importância do estudo levantado não só no âmbito da segurança pública e combate ao crime, como também no âmbito social, convívio entre as espécies e na manutenção dos benefícios mútuos existentes nessas relações algumas medidas para amenizar os efeitos do aumento das concentrações de cortisol como o oferecimento de um período de descanso após o confinamento e o transporte dos cães, promovendo períodos com interações de brinquedos; diminuir o tempo de espera pelo embarque enquanto confinados, diminuindo assim o tempo de confinamento nas caixas de transporte; aumentar o período de atividades e treinamentos desses animais no cotidiano ou mesmo em períodos que antecedem o confinamento de transporte, não só como forma de reforço do condicionamento, mas como uma forma de mantêlos mais ativos e exaustivos, podendo influenciar de forma contrária à demonstrada no estudo, os 
efeitos do confinamento para o transporte, ou seja, enfrentando o confinamento nas caixas como uma forma de descanso.

Por conseguinte, é importante mencionar a dimensão que o presente estudo apresenta, podendo abrir caminhos para inúmeras novas pesquisas dos parâmetros comportamentais e fisiológicos de cães policiais buscando conhecer mais a fundo, por exemplo, as influências da termorregulação na olfação, ou as alterações fisiológicas sofridas por esses animais em condições de estresse ou trabalhos contínuos que possam afetar o desempenho dos trabalhos de busca e detecção, ou ainda, buscar conhecer as diferenças de personalidades e qualidades intrínsecas das raças que influenciam na seleção de sujeitos aptos ao trabalho policial. Enfim, são inúmeras as pesquisas que ainda podem ser elaboradas nesse campo de atuação do trabalho de cães policiais.

\section{Referências Bibliográficas}

Adams G.J. \& Johnson K.G. 1994. Sleep, work, and the effects of shift work in drug detector dogs Canis familiaris. Applied Animal Behaviour Science 41, 115-26.

Ahrens F., Knies K., Schneider M., Köhler F. \& Erhard M.H. 2005. Influence of different training and outdoor conditions on plasma histamine and cortisol concentrations in search-and-rescue dogs. Inflammation Research 54, S34-S5.

Bateson P., Barker D., Clutton-Brock T., Deb D., D'udine B., Foley R.A., Gluckman P., Godfrey K., Kirkwood T. \& Lahr M.M. 2004. Developmental plasticity and human health. Nature 430, 419.

Beerda B., Schilder M.B.H., Janssen N.S.C.R.M. \& Mol J.A. 1996. The use of saliva cortisol, urinary cortisol, and catecholamine measurements for a noninvasive assessment of stress responses in dogs. Hormones and behavior 30, 272-9.

Bergeron R., Scott S.L., Émond J.-P., Mercier F., Cook N.J. \& Schaefer A.1.L. 2002. Physiology and behavior of dogs during air transport. Canadian Journal of Veterinary Research 66, 211-6.

Broom D.M. \& Johnson K.G. 1993. Stress and animal welfare. Springer Science \& Business Media, London.
Freitas E.G. \& Nishida S.M. 2007. Métodos de estudo do comportamento animal. Comportamento Animal 1, 39-64.

Gazit I. \& Terkel J. 2003. Explosives detection by sniffer dogs following strenuous physical activity. Applied Animal Behaviour Science 81, 149-61.

Haubenhofer D.K. \& Kirchengast S. 2006. Physiological arousal for companion dogs working with their owners in animal-assisted activities and animal-assisted therapy. Journal of Applied Animal Welfare Science 9, 165-72.

Haverbeke A., Diederich C., Depiereux E. \& Giffroy J.M. 2008. Cortisol and behavioral responses of working dogs to environmental challenges. Physiology \& behavior 93, 59-67.

Jezierski T., Walczak M. \& Górecka A. 2008. Information-seeking behaviour of sniffer dogs during match-to-sample training in the scent lineup. Polish Psychological Bulletin 39, 7180.

LeDoux J. 2003. The emotional brain, fear, and the amygdala. Cellular and molecular neurobiology 23, 727-38.

Lefebvre D., Diederich C., Delcourt M. \& Giffroy J.-M. 2007. The quality of the relation between handler and military dogs influences efficiency and welfare of dogs. Applied Animal Behaviour Science 104, 49-60.

Lindsay S. 2000. Handbook of applied dog behavior and training. Blackwell, Iowa, USA.

Lit L., Schweitzer J.B. \& Oberbauer A.M. 2011. Handler beliefs affect scent detection dog outcomes. Animal cognition 14, 387-94.

Lovett S. 1992. Explosive search dogs. In: Proceedings of the First International Symposium on Explosive Detection Technology (ed. by Khan SM.) FAA Technical Center, Atlantic City, New Jersey.

Montanha J.C., Silva S.L. \& Boere V. 2009. Comparação das concentrações de cortisol salivar em onças-pintadas criadas em cativeiro com diferenças de exposição ao público. Ciência Rural 39, 1745-51.

Moody J.A., Clark L.A. \& Murphy K.E. 2006. Working dog: History and applications. In: The dog and its genome (eds. by Ostrander EA, Giger U \& Lindblad-Toh K). Cold Spring Harbor Laboratory Press, USA.

Morrison E.E. 2000. Canine detection of illict drugs: Sensory apparatus technology. Morrison, E. E. 2000. Canine detection of 
illicit drugs: sensory apparatus technology, Auburn University, USA.

Santana J.A., Castro I.P. \& Almeida L.P. 2010. Caracterização do convívio entre o proprietário e o cão atendido no hospital veterinário da Universidade Federal de Uberlândia. PUBVET 4, 752-8.

Schatz S. \& Palme R. 2001. Measurement of faecal cortisol metabolites in cats and dogs: a non-invasive method for evaluating adrenocortical function. Veterinary research communications 25, 271-87.

Strasser A., Niedermüller H., Hofecker G. \& Laber G. 1993. The effect of aging on laboratory values in dogs. Transboundary and Emerging Diseases 40, 720-30.

Wilson D.A., Best A.R. \& Sullivan R.M. 2004. Plasticity in the olfactory system: lessons for the neurobiology of memory. The Neuroscientist 10, 513-24.

Article History:

Received 15 February 2018

Accepted 20 March 2018

Available online 31 May 2018

License information: This is an open-access article distributed under the terms of the Creative Commons Attribution License 4.0, which permits unrestricted use, distribution, and reproduction in any medium, provided the original work is properly cited. 